\title{
Reverse Shoulder Arthroplasty for the treatment of Proximal humeral fractures in the Elderly (ReShAPE trial) : study protocol for a multicentre combined randomised controlled and observational trial
}

\author{
Geoffrey C. S. Smith ${ }^{1,12^{*}}$, Ed Bateman², Ben Cass ${ }^{3}$, Maurizio Damiani ${ }^{4}$, Wade Harper ${ }^{5}$, Hugh Jones ${ }^{1}$, David Lieu', \\ Jeff Petchell ${ }^{7}$, Minas Petrelis ${ }^{8}$, Kalman Piper ${ }^{9}$, Doron Sher ${ }^{10}$, Christopher J. Smithers ${ }^{7}$, John Trantalis ${ }^{10}$, \\ Sindy Vrancic ${ }^{4}$ and lan A. Harris ${ }^{11}$
}

\begin{abstract}
Background: Proximal humeral fractures are common in older patients. The majority are minimally displaced and are associated with good outcomes after nonoperative treatment. Poorer outcomes are associated with displaced, multipart fractures. There is no clear benefit from surgical fracture fixation compared to nonoperative treatment. Replacement of the fractured humeral head with a hemiarthroplasty is another treatment option, but has not been shown to be clearly superior to nonoperative treatment or internal fixation. Recently, reverse total shoulder arthroplasty has been used to treat these fractures, particularly in the older population with several case series demonstrating good outcomes. No comparative trial has been performed to test the effectiveness of reverse total shoulder arthroplasty against nonoperative treatment.

Methods/design: ReShAPE (Reverse Shoulder Arthroplasty for the treatment of Proximal humeral fractures in the Elderly) is a multicenter combined randomized and observational study. The primary objective is to compare pain and function 12 months post fracture using the American Shoulder and Elbow Society (ASES) score in patients aged 70 years or older with three- and four-part proximal humeral fractures treated by either reverse shoulder arthroplasty or nonoperative treatment. Secondary outcome measures will include the DASH (Disability of the Arm, Shoulder and Hand) score, the EQ-5D (EuroQol Health Survey), the EQ-VAS, pain, radiological parameters and complications.
\end{abstract}

Discussion: The study will assess the effectiveness of reverse shoulder arthroplasty for complex proximal humeral fractures and thereby guide treatment of a common injury in the older population.

Trial registration: World Health Organization Universal Trial Number (WHO UTN): U1111-1180-5452. Registered on 10 March 2016.

Australian and New Zealand Clinical Trials Registry (ANZCTR): 12616000345482. Registered on 16 March 2016.

Keywords: Fracture, Proximal humerus, Treatment, Surgery, Arthroplasty, Reverse shoulder arthroplasty

\footnotetext{
* Correspondence: gcssmith@icloud.com

${ }^{1}$ St. George and Sutherland Hospitals, Grey St, Kogarah, NSW, Australia

${ }^{12}$ Sydney Orthopaedic Trauma and Reconstructive Surgery, 5/19 Kensington

St, Kogarah, Sydney, NSW 2217, Australia

Full list of author information is available at the end of the article
} 


\section{Background}

Humeral neck fractures account for $5 \%$ of fractures of the appendicular skeleton [1] and are the third commonest osteoporotic fracture [2] occurring with an incidence of 6.6 per 1000 person years [3]. There is a unipolar age distribution with most occurring in the older independent population with osteoporosis who fall from a standing height [4]. This incidence is set to increase in the next 20 years as a result of population growth and an aging population [5]. Forty-nine to $85 \%$ of proximal humeral fractures are minimally displaced and are usually treated nonoperatively with most having a good outcome regardless of comminution $[4,6,7]$. The poorer outcomes associated with displaced, multipart fractures has led surgeons to investigate operative alternatives $[3,8,9]$. Internal fixation with locking plates, which have some advantages in osteoporotic bone, have been subject to randomized trials, but have not been shown to improve outcome over nonoperative treatment [10-13]. Replacement of the fractured humeral head (hemiarthroplasty) is another treatment option, but has not been shown to be clearly superior to nonoperative treatment or plate fixation $[14,15]$.

Recently, reverse total shoulder arthroplasty has been used to treat these fractures, with several case series published [16-20]. This prosthetic design negates the effects of tuberosity malunion and nonunion that are common after internal fixation or hemiarthroplasty by creating a mechanical advantage for the deltoid muscle to allow active forward elevation and abduction. Studies comparing reverse total shoulder arthroplasty to hemiarthroplasty for the treatment of proximal humeral fractures have shown improved pain scores and functional outcomes after reverse shoulder arthroplasty [21, 22]. The use of reverse shoulder arthroplasty for the treatment of proximal humeral fractures is increasing [23]. Reverse shoulder arthroplasty has been reported to have a high complication rate including instability, loosening, poor rotation and radiological notching [24]. The risk of complications and prosthetic longevity limits the use of reverse shoulder arthroplasty in young patients with most prostheses being inserted in patients aged over 65 years [25].

\section{Methods/design}

\section{Study objectives}

We aim to test the hypothesis that in patients aged 70 years and over with three- or four-part fractures of the humeral neck, treatment with reverse total shoulder arthroplasty will result in improved shoulder pain and function 2 years post injury compared to nonoperative treatment.

\section{Study design}

The study is a combined multicenter randomized controlled trial (RCT) combined with an observational cohort study. Eleven centers in Australia will participate. The study Consolidated Standards of Reporting Trials (CONSORT) flowchart is provided in Fig. 1. The SPIRIT Checklist is provided in Additional file 1 and the Standard Protocol Items: Recommendations for Interventional Trials (SPIRIT) diagram is provided in Fig. 2

\section{Ethics}

The recruiting sites in alphabetical order are: The Canberra Hospital, Concord Hospital, Gosford Hospital, John Hunter Hospital, Liverpool Hospital, Prince of Wales Hospital, Royal North Shore Hospital, Royal Prince Alfred Hospital, St. George Hospital, Sutherland Hospital and Westmead Hospital.

The study is registered with the Australasian and New Zealand Clinical Trials Registry (12616000345482) and the World Health Organization Universal Trail Registry (U1111-1180-5452)

\section{Study population}

Patients will be considered for the study if they are 70 years of age or older, medically fit for surgery, have a three- or four-part proximal humerus fracture according to Neer's classification [6] (Table 1), present within 28 days after injury, and are available for follow-up for 24 months.

Patients will be excluded if they are unable to provide consent due to limited proficiency in English or cognitive impairment (three or more errors on the Mini Mental Status Examination 6-item screening tool) [26], live in a residential aged care facility, have a pre-existing injury or condition of the affected side which severely affects the movement of the shoulder, a pathological fracture, other injury to the same upper limb requiring surgery, an open fracture, a fracture-dislocation or headsplitting fracture, a glenoid fracture, gross fracture displacement (no bony contact between humeral shaft and articular segment) or an axillary nerve palsy.

\section{Recruitment}

Patients will be approached on presentation to a participating institution either in the emergency department or in the outpatient setting. Patients aged 70 years and over with a displaced proximal humerus fracture with tuberosity involvement will undergo a computed tomography (CT) scan with $3 \mathrm{D}$ reconstructions to assess the fracture configuration and to assess the number of fracture parts according to Neer's classification [6] (Table 1). Linear measurements will be made with digital calipers. The normal neck shaft angle will be assumed to be $130^{\circ}$. Verbal consent will be requested to allow questioning to assess eligibility and to collect identifiable data. The fracture type will be screened against the inclusion criteria by two of the principal investigators. Patients who satisfy 


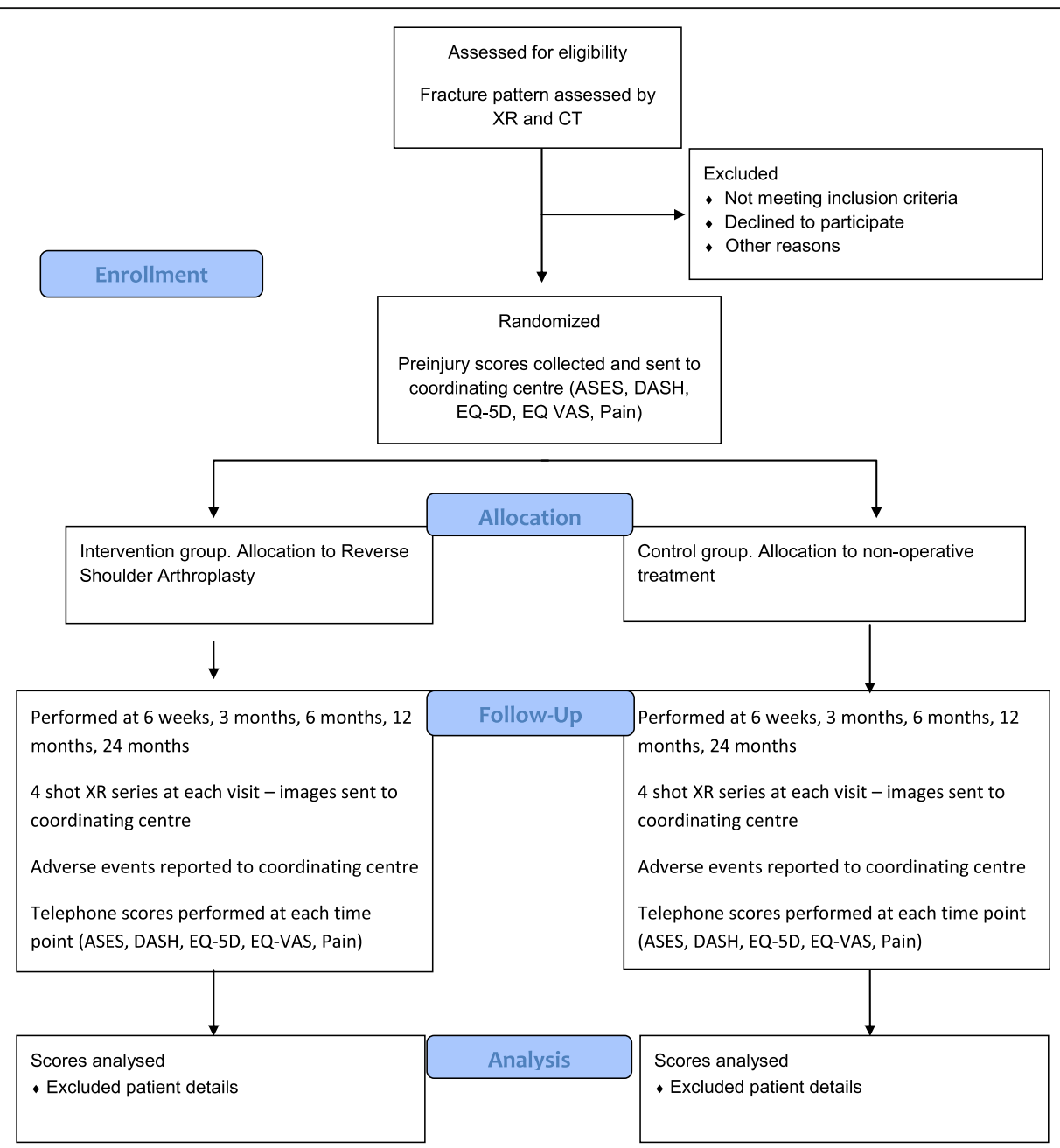

Fig. 1 Consolidated Standards of Reporting Trials (CONSORT) flowchart

the study criteria will be invited to participate in the study and will receive a Participant Information Sheet (see Additional file 1). Patients who are willing to participate in the randomized trial will be asked to sign a Consent Form. Basic demographic and radiographic data will be collected for both groups to assess independent variables (age, gender, fracture type, diabetes (yes/no), smoking status (current/non), baseline shoulder function (American Shoulder and Elbow Society (ASES) score, Disability of the Arm, Shoulder and Hand (DASH) score) and baseline health (EuroQol Health Survey 5 dimensions (EQ-5D) and EuroQol Visual Analog Scale (EQ-VAS)). Patients who are unwilling to participate in the randomized trial will be offered participation in the observational study and will be provided with a separate Participant Information Sheet and Consent Form.

\section{Randomization}

Randomization will occur immediately after consent has been gained. Randomization will occur by using a central, telephone-based automated randomization service with each site having its own access code (provided by the NHMRC Clinical Trials Center). Randomization will be stratified by site and will use minimization to adjust for gender and age (70-80 years, over 80 years).

\section{Interventions}

Participants who are randomized to surgery will be treated by insertion of a reverse total shoulder arthroplasty within 28 days of the date of injury. Surgical technique (approach, version, component fixation and prosthesis choice) will be left to the discretion of the treating surgeon. The glenosphere will be placed low, avoiding superior tilt. The tuberosities will be repaired using nonabsorbable sutures. Postoperatively, the arm will be placed in a shoulder immobilizer and patients will be instructed on elbow, wrist and hand exercises to commence immediately. Two weeks postoperatively, pendular exercises and passive flexion to $90^{\circ}$ and passive external rotation to neutral will be commenced. Unrestricted passive and active 


\begin{tabular}{|c|c|c|c|c|c|c|c|c|}
\hline \multirow[b]{3}{*}{ TIMEPOINT ${ }^{* *}$} & \multicolumn{8}{|c|}{ STUDY PERIOD } \\
\hline & \multirow{2}{*}{$\begin{array}{c}\text { Enrolment } \\
-t_{1}\end{array}$} & \multirow{2}{*}{$\begin{array}{c}\text { Allocation } \\
0\end{array}$} & \multicolumn{5}{|c|}{ Post-allocation } & \multirow{2}{*}{$\begin{array}{r}\text { Close-out } \\
2 \text { years }\end{array}$} \\
\hline & & & $\begin{array}{c}0 \\
\text { wee } \\
k s\end{array}$ & $\begin{array}{c}6 \\
\text { wee } \\
k s\end{array}$ & $\begin{array}{c}3 \\
\text { mon } \\
\text { ths }\end{array}$ & $\begin{array}{c}6 \\
\text { mon } \\
\text { ths }\end{array}$ & $\begin{array}{c}1 \\
\text { year }\end{array}$ & \\
\hline \multicolumn{9}{|l|}{ ENROLMENT: } \\
\hline \multirow{3}{*}{$\begin{array}{l}\text { Eligibility screen } \\
\text { Informed consent } \\
\text { Allocation }\end{array}$} & $x$ & & & & & & & \\
\hline & $x$ & & & & & & & \\
\hline & & $x$ & & & & & & \\
\hline \multicolumn{9}{|l|}{ INTERVENTIONS: } \\
\hline \multicolumn{9}{|l|}{$\begin{array}{r}\text { Reverse shoulder } \\
\text { arthroplasty }\end{array}$} \\
\hline \multicolumn{9}{|l|}{$\begin{array}{r}\text { Non-operative } \\
\text { treatment }\end{array}$} \\
\hline \multicolumn{9}{|l|}{$\begin{array}{r}\text { [List other study } \\
\text { groups] }\end{array}$} \\
\hline \multicolumn{9}{|l|}{ ASSESSMENTS: } \\
\hline \multicolumn{9}{|l|}{$\begin{array}{r}\text { Age, Gender, } \\
\text { Fracture type, DM, } \\
\text { Smoking, ASES, } \\
D A S H, E Q-5 D, E Q- \\
\text { VAS }\end{array}$} \\
\hline \multirow{2}{*}{$\begin{array}{r}A S E S \\
\text { DASH, EQ-5D, EQ- } \\
V A S, \text { Pain VAS, } \\
\text { radiological } \\
\text { scores. }\end{array}$} & & & & $X$ & $X$ & $X$ & $x$ & $x$ \\
\hline & & & $x$ & $\mathrm{X}$ & $\mathrm{X}$ & $X$ & $x$ & $\mathrm{X}$ \\
\hline
\end{tabular}

Fig. 2 Standard Protocol Items: Recommendations for Interventional Trials (SPIRIT) diagram

assisted exercises will be allowed, graduating to active mobilization (as tolerated) at 6 weeks post surgery. Resisted range of motion exercises will be allowed after 12 weeks. A minimum of five physiotherapy contacts within 3 months of treatment will be provided.

Patients randomized to nonoperative treatment will be placed in a shoulder immobilizer. Elbow wrist and hand exercises will be allowed immediately. After

Table 1 Neer's classification of proximal humeral fractures and radiological inclusion criteria

Potential fracture "parts" (Neer) Head (H), Shaft (S), Lesser tuberosity(LT), Greater tuberosity (GT)

$\begin{array}{cc}\text { Definition of a "part" (Neer) } & >1 \mathrm{~cm} \text { displacement or }>45^{\circ} \text { rotation. } \\ & \text { Normal neck shaft angle } 130^{\circ} \\ \text { Radiological inclusion criteria } & \text { 3-part fractures: } \\ & H+\text { GT, S and LT } \\ H+L T, S \text { and GT } \\ \text { H, LT + GT and S } \\ \text { H, GT, LT + S } \\ \text { H, LT, GT + S } \\ \text { 4-part fractures: } \\ \text { H, LT, GT and S }\end{array}$

2 weeks, pendular exercises and passive flexion to $90^{\circ}$ and passive external rotation to neutral will be commenced. Unrestricted passive and active assisted exercises will be allowed graduating to active mobilization (as tolerated) at 6 weeks. Resisted range of motion exercises will be allowed after 12 weeks. A minimum of five physiotherapy contacts within 3 months of treatment will be provided.

\section{Parallel observational cohort study}

Patients who do not consent to be randomized will be offered participation in the observational arm of the study. Their treatment will consist of the same two treatment options as the RCT arm. Treatment will be decided by patient and surgeon preference as per usual practice at each institution. Treatment protocols, followup and outcome measures will be the same as the randomized arm. The outcome of this arm of the trial will be analyzed separately. The inclusion of the observational cohort will inform the generalizability of the randomized trial. 


\section{Outcome measures}

Participants will be followed up at 6 weeks, 3 months, 6 months, 1 year, 2 years, 5 years and 10 years. The outcome measures listed below will be assessed at 6 weeks, 3 months, 6 months, 1 year, 2 years, 5 years and 10 years.

The primary outcome will be the ASES (American Shoulder and Elbow Society) standardized shoulder assessment score patient self-reported section at 24 months [27]. This scoring system consists of two dimensions: pain and activities of daily living, which are both equally weighted giving a total score out of 100, with higher scores indicating less pain and better function. It has been shown to be reliable, valid and responsive in shoulder arthroplasty and fracture [28, 29]. Secondary outcome measures will include DASH (Disability of the Arm, Shoulder and Hand) score [30], the EQ-5D and the EQ-VAS, pain (verbal numerical rating scale, 0-10 points), radiological parameters and complications (repeat shoulder surgery, readmission, infection requiring treatment, neurological deficit, dislocation, death). Radiographs will be performed at each follow-up and will include a four-radiograph series: an AP view in neutral and $30^{\circ}$ external rotation, and a transthoracic scapula "Y" view and axillary view. Radiographic parameters that will be assessed are: healing of the tuberosities (nonunion or resorption will be considered failures to heal), position of the tuberosities, scapula notching (according to the Sirveaux classification system [31]) and loosening and alignment (coronal and sagittal plane for patients treated nonoperatively). All radiographs will be assessed by two independent observers independently. In case of discrepancy the scoring will be decided by discussion.

\section{Sample size calculation}

Case series documenting the outcome after reverse shoulder arthroplasty have shown a standard deviation (SD) of 13 in the ASES score. The minimum important clinical difference (MICD) in ASES scores has been estimated to be 6.4-18 [28,32]. We consider a difference of 10 to be necessary in order to justify the additional costs and risks of surgery. A total of 60 patients (30 in each group) will provide $80 \%$ power to detect a MICD of 10 points at a significance of 0.05 . We aim to recruit 72 patients to allow for $17 \%$ loss to follow-up. The ASES score after reverse shoulder arthroplasty has been reported to be 68-86 (higher score being better) [33]. In a paper reporting the outcomes of two-, three- and four-part fractures (our study proposal is limited to three- and four-part fractures) treated nonsurgically the mean ASES score was 82.5 [13].

\section{Statistical analysis}

Differences in baseline characteristics (i.e., intrinsic and injury-related variables) between both intervention groups will be assessed using the Student's $t$ test (parametric continuous data), the Mann-Whitney $U$ test (nonparametric continuous data) or the chi-square test (categorical data).

Unadjusted analysis by intention-to-treat will be performed to test the difference in primary and secondary outcomes between the intervention groups. Student's $t$ test (parametric continuous data), the Mann-Whitney $U$ test (nonparametric continuous data) or the chi-square analysis (categorical data) will be used. A $p$ value $<0.05$ (two-sided tests) will be taken as threshold of statistical significance.

The observational arm will be analyzed separately, comparing the same treatment groups against the same outcomes using multivariable linear regression to adjust for potential confounders (age, sex, handedness, smoking, fracture type).

\section{Monitoring and quality assurance}

Adverse events to be recorded are repeat shoulder surgery, readmission, infection requiring treatment, neurological deficit, dislocation and death. All adverse outcomes will be recorded centrally.

Data will be collected by local site investigators and study documents will be submitted securely (scanned and emailed) to the project manager at the administering institution. Identifying data will be transferred separately from any study information and stored separately to the study database. All research documentation will be labelled with a unique person number as an identifier and stripped of any potentially identifiable information. Data will be stored in password-protected computers and locked filing cabinets as required within the administering institution

\section{Blinding}

Study investigators, surgeons and participants will not be blinded to the treatment group due to the nature of the comparators (surgery versus no surgery). The primary outcome (ASES score) and secondary outcome scores will be collected by blinded researchers by telephone at all time points. The statistician will be blinded to the treatment group

\section{Discussion}

The optimal treatment of three- and four-part fractures of the proximal humerus in older people has not yet been established. Operative treatment in the form of internal fixation or hemiarthroplasty has not been shown to be beneficial over nonoperative treatment. The design of the reverse shoulder arthroplasty has theoretical advantages in the treatment of these difficult fractures and several case series have shown good outcomes after reverse shoulder arthroplasty. However, there are no prospective RCTs comparing reverse shoulder arthroplasty 
to nonoperative treatment of displaced proximal humeral fractures in older people.

The ReShAPE trial will compare these treatment modalities and will guide future treatment for this common injury, potentially changing current practice.

The use of multiple different centers, variability in selection of surgical technique (approach, version, component fixation and prosthesis choice) and an observational cohort will increase the generalizability of the results. Randomization will occur only after review of the scans and after eligibility has been determined; therefore, making the two groups comparable and reducing selection bias.

\section{Trial status}

The ReShAPE trial commenced recruitment in March 2016 but this has not yet been completed.

\section{Additional file}

Additional file 1: SPIRIT 2013 Checklist: recommended items to address in a clinical trial protocol and related documents*. (DOC $121 \mathrm{~kb}$ )

\section{Abbreviations}

ASES: American Shoulder and Elbow Society; DASH: Disability of the Arm, Shoulder and Hand

\section{Acknowledgements}

The initial setup of this trial was supported by a grant from the St. George Hospital Orthopaedic Department.

\section{Funding}

A grant was obtained from the Australian Orthopaedic Association to support a study coordinator.

The funding bodies are independent of the design of the study and collection, analysis and interpretation of data and in writing the manuscript.

\section{Availability of data and materials}

The datasets used and/or analyzed during the current study are available from the corresponding author on reasonable request.

\section{Authors' contributions}

GS developed the trial and drafted the manuscript, will act as chief investigator, will act as principal investigator at two of the sites and will perform the surgical procedures and participate in patient inclusion and assessment. $\mathrm{IH}$ developed the trial and drafted the manuscript. WH will act as principal investigator, will perform the surgical procedures and will participate in patient inclusion and assessment. DL will act as principal investigator, will perform the surgical procedures and will participate in patient inclusion and assessment. CS will act as principal investigator, will perform the surgical procedures and will participate in patient inclusion and assessment. JT will act as principal investigator, will perform the surgical procedures and will participate in patient inclusion and assessment. BC will act as principal investigator, will perform the surgical procedures and will participate in patient inclusion and assessment. MP will act as principal investigator, will perform the surgical procedures and will participate in patient inclusion and assessment. EB will act as principal investigator, will perform the surgical procedures and will participate in patient inclusion and assessment. MD will act as principal investigator, will perform the surgical procedures and will participate in patient inclusion and assessment. KP will act as principal investigator, will perform the surgical procedures and will participate in patient inclusion and assessment. HJ will perform the surgical procedures and will participate in patient inclusion and assessment. SV will perform the surgical procedures and will participate in patient inclusion and assessment. JP will perform the surgical procedures and will participate in patient inclusion and assessment. DS will perform the surgical procedures and will participate in patient inclusion and assessment. All authors have read and approved the manuscript.

\section{Competing interests}

The authors declare that they have no competing interests.

\section{Consent for publication}

Not applicable.

\section{Ethics approval and consent to participate}

ReShAPE has been approved by South Eastern Sydney Local Health District Human Research Ethics Committee (Reference number 15/029 HREC/15/ POWH/98).

Randomization will occur only after written consent has been gained.

\section{Author details}

${ }^{1}$ St. George and Sutherland Hospitals, Grey St, Kogarah, NSW, Australia. ${ }^{2}$ Gosford District Hospital, Holden St, Gosford, NSW, Australia. ${ }^{3}$ Royal North Shore Hospital, Reserve Rd, St. Leonards, NSW, Australia. ${ }^{4}$ The Canberra Hospital, 51 Jardine St, Kingston ACT, Australia. ${ }^{5}$ Prince of Wales Hospital, Barker St, Randwick, NSW, Australia. 'Liverpool Hospital, Locked Bag 7103, Liverpool, NSW, Australia. ${ }^{7}$ Royal Prince Alfred Hospital, Missenden Rd, Camperdown, NSW, Australia. ${ }^{8}$ John Hunter Hospital, Lookout Rd, New Lambton Heights, NSW, Australia. ' Westmead Hospital, Hawkesbury Rd and Darcy Rd, Westmead, NSW, Australia. ${ }^{10}$ Concord Hospital, Hospital Road, Concord, NSW, Australia. ${ }^{11}$ Whitlam Orthopaedic Research Centre, Ingham Institute for Applied Medical Research, South Western Sydney Clinical School, UNSW Australia, Liverpool, NSW, Australia. ${ }^{12}$ Sydney Orthopaedic Trauma and Reconstructive Surgery, 5/19 Kensington St, Kogarah, Sydney, NSW 2217, Australia.

Received: 4 May 2016 Accepted: 6 February 2017

Published online: 28 February 2017

\section{References}

1. Court-Brown CM, Caesar B. Epidemiology of adult fractures: a review. Injury. 2006:37(8):691-7.

2. Calvo E, Morcillo D, Foruria AM, Redondo-Santamaria E, Osorio-Picorne F, Caeiro JR. Nondisplaced proximal humeral fractures: high incidence among outpatient-treated osteoporotic fractures and severe impact on upper extremity function and patient subjective health perception. J Shoulder Elb Surg. 2011;20(5):795-801

3. Lanting B, MacDermid J, Drosdowech D, Faber KJ. Proximal humeral fractures: a systematic review of treatment modalities. J Shoulder Elb Surg. 2008;17(1):42-54.

4. Court-Brown CM, Garg A, McQueen MM. The epidemiology of proximal humeral fractures. Acta Orthop Scand. 2001;72(4):365-71.

5. Kannus $P$, Palvanen M, Niemi S, Parkkari J, Jarvinen M, Vuori I. Osteoporotic fractures of the proximal humerus in elderly Finnish persons: sharp increase in 1970-1998 and alarming projections for the new millennium. Acta Orthop Scand. 2000;71(5):465-70.

6. Neer 2 nd CS. Displaced proximal humeral fractures. I. Classification and evaluation. J Bone Joint Surg Am. 1970;52(6):1077-89.

7. Gaebler C, McQueen MM, Court-Brown CM. Minimally displaced proximal humeral fractures: epidemiology and outcome in 507 cases. Acta Orthop Scand. 2003;74(5):580-5.

8. Nho SJ. Management of proximal humeral fractures based on current literature. J Bone Joint Surg Am. 2007;89 Suppl 3:44.

9. Murray IR, Amin AK, White TO, Robinson CM. Proximal humeral fractures: current concepts in classification, treatment and outcomes. J Bone Joint Surg (Br). 2011;93(1):1-11.

10. Olerud P, Ahrengart L, Ponzer S, Saving J, Tidermark J. Internal fixation versus nonoperative treatment of displaced 3-part proximal humeral fractures in elderly patients: a randomized controlled trial. J Shoulder Elb Surg. 2011;20(5):747-55.

11. Fjalestad T, Hole MO, Hovden IAH, Blucher J, Stromsoe K. Surgical treatment with an angular stable plate for complex displaced proximal humeral fractures in elderly patients: a randomized controlled trial. J Orthop Trauma. 2012;26(2):98-106 
12. Rangan A, Handoll H, Brealey S, Jefferson L, Keding A, Corbacho Martin B, Goodchild L, Chuang L-H, Hewitt C, Torgerson D. Surgical vs nonsurgical treatment of adults with displaced fractures of the proximal humerus. 2015; 313(10):1037-11. Available from: http://jamanetwork.com/journals/jama/ fullarticle/2190987.

13. Sanders RJ, Thissen LG, Teepen JC, van Kampen A, Jaarsma RL. Locking plate versus nonsurgical treatment for proximal humeral fractures: better midterm outcome with nonsurgical treatment. J Shoulder Elb Surg. 2011;20(7):1118-24.

14. Boons HW, Goosen JH, van Grinsven S, van Susante JL, van Loon CJ. Hemiarthroplasty for humeral four-part fractures for patients 65 years and older: a randomized controlled trial. Clin Orthop Relat Res. 2012;470(12):3483-91.

15. Olerud P, Ahrengart L, Ponzer S, Saving J, Tidermark J. Hemiarthroplasty versus nonoperative treatment of displaced 4-part proximal humeral fractures in elderly patients: a randomized controlled trial. J Shoulder Elb Surg. 2011;20(7):1025-33.

16. Ross M, Hope B, Stokes A, Peters SE, McLeod I, Duke PFR. Reverse shoulder arthroplasty for the treatment of three-part and four-part proximal humeral fractures in the elderly. J Shoulder Elbow Surg. 2015;24(2):215-22. doi: 10. 1016/j.jse.2014.05.022. Epub 2014 Aug 29.

17. Lenarz C, Shishani Y, McCrum C, Nowinski RJ, Edwards TB, Gobezie R. Is reverse shoulder arthroplasty appropriate for the treatment of fractures in the older patient?: early observations. Clin Orthop Relat Res. 2011;469(12):3324-31.

18. Cazeneuve J-F, Cristofari D-J. The reverse shoulder prosthesis in the treatment of fractures of the proximal humerus in the elderly. J Bone Joint Surg (Br). 2010;92(4):535-9.

19. Cazeneuve J-F, Cristofari D-J. Long term functional outcome following reverse shoulder arthroplasty in the elderly. Orthop Traumatol Surg Res. 2011;97(6):583-9.

20. Bufquin T, Hersan A, Hubert L, Massin P. Reverse shoulder arthroplasty for the treatment of three- and four-part fractures of the proximal humerus in the elderly: a prospective review of 43 cases with a short-term follow-up. J Bone Joint Surg (Br). 2007;89(4):516-20.

21. Cuff D. Reverse shoulder arthroplasty for the treatment of rotator cuff deficiency. J Bone Joint Surg Am. 2012;94(21):1996.

22. Gallinet D, Clappaz P, Garbuio P, Tropet Y, Obert L. Three or four parts complex proximal humerus fractures: hemiarthroplasty versus reverse prosthesis: a comparative study of 40 cases. Orthop Traumatol Surg Res. 2009;95(1):48-55.

23. Acevedo DC, Vanbeek C, Lazarus MD, Williams GR, Abboud JA. Reverse shoulder arthroplasty for proximal humeral fractures: update on indications, technique, and results. J Shoulder Elb Surg. 2014;23(2):279-89.

24. Cheung E, Willis M, Walker M, Clark R, Frankle MA. Complications in reverse total shoulder arthroplasty. J Am Acad Orthop Surg. 2011;19(7):439-49.

25. Handoll HHG, Brorson S. Interventions for treating proximal humeral fractures in adults (Review). Cochrane Database Syst Rev. 2015;(11): CD000434. doi:10.1002/14651858.CD000434.pub4.

26. Callahan CM, Unverzagt FW, Hui SL, Parsons AJ, Hendrie HC. Six-item screener to identify cognitive impairment among potential subjects for clinical research. 2002:40(9):771-81.

27. Richards RR, An KN, Bigliani LU, Friedman RJ, Gartsman GM, Gristina AG, et al. A standardized method for the assessment of shoulder function. J Shoulder Elb Surg. 1994;3(6):347-52.

28. Michener LA, McClure PW, Sennett BJ. American Shoulder and Elbow Surgeons Standardized Shoulder Assessment Form, patient self-report section: reliability, validity, and responsiveness. J Shoulder Elb Surg. 2002; 11(6):587-94.

29. Roy J-S, MacDermid JC, Woodhouse LJ. Measuring shoulder function: a systematic review of four questionnaires. Arthritis Rheum. 2009;61 (5):623-32.

30. Hudak PL, Amadio PC, Bombardier C. Development of an upper extremity outcome measure: the DASH (disabilities of the arm, shoulder and hand) [corrected]. The Upper Extremity Collaborative Group (UECG). Am J Ind Med. 1996;29(6):602-8.

31. Sirveaux F, Favard L, Oudet D, Huquet D, Walch G, Mole D. Grammont inverted total shoulder arthroplasty in the treatment of glenohumeral osteoarthritis with massive rupture of the cuff. Results of a multicentre study of 80 shoulders. J Bone Joint Surg (Br). 2004;86(3):388-95.

32. Tashjian RZ. Minimal clinically important differences in ASES and Simple Shoulder Test Scores after nonoperative treatment of rotator cuff disease. J Bone Joint Surg Am. 2010;92(2):296.

33. Mata-Fink A, Meinke M, Jones C, Kim B, Bell J-E. Reverse shoulder arthroplasty for treatment of proximal humeral fractures in older adults: a systematic review. J Shoulder Elb Surg. 2013;22(12):1737-48.

\section{Submit your next manuscript to BioMed Central and we will help you at every step:}

- We accept pre-submission inquiries

- Our selector tool helps you to find the most relevant journal

- We provide round the clock customer support

- Convenient online submission

- Thorough peer review

- Inclusion in PubMed and all major indexing services

- Maximum visibility for your research

Submit your manuscript at www.biomedcentral.com/submit 